\title{
Inelastic photon-neutrino interactions using an effective Lagrangian
}

\author{
A. Abada, ${ }^{*}$ J. Matias ${ }^{\dagger}$ and R. Pittau ${ }^{\ddagger}$ \\ Theory Division, CERN, CH-1211 Geneva 23, Switzerland \\ (Received 19 June 1998; published 2 December 1998)
}

\begin{abstract}
We justify the feasibility of substituting a photon leg by a neutrino current in the Euler-Heisenberg Lagrangian to obtain an effective Lagrangian for the process $\gamma \nu \rightarrow \gamma \gamma \nu$ and its crossed reactions. We establish the link between these processes and the four-photon scattering in both the standard model and the effective theory. As an application, we compute the processes $\gamma \nu \rightarrow \gamma \gamma \nu$ and $\gamma \gamma \rightarrow \gamma \nu \bar{\nu}$, give their polarized cross sections, and show how to use the $\gamma \gamma \rightarrow \gamma \gamma$ results as a check. We settle the question about the disagreement between two computations in the literature concerning the reaction $\gamma \gamma \rightarrow \gamma \nu \bar{\nu}$. [S0556-2821(99)03301-9]
\end{abstract}

PACS number(s): 13.15.+g, 13.88.+e, 14.70.Bh, 95.30.Cq

\section{INTRODUCTION}

Processes involving neutrinos play an important role in astrophysics. For example, during the evolution of a star, neutrino emission carries energy away from the entire volume of the star, while, because of short range interactions, the compact (dense) central part is opaque for photons, which remain trapped inside. In that sense, contrary to the neutrino, the photon is an "amnesic" particle.

In particular, despite their small cross section, low-energy photon-neutrino processes are potentially of interest in stellar evolution as well as in cosmology. Typical examples are the processes

$$
\begin{aligned}
& \gamma \nu \rightarrow \gamma \nu \\
& \gamma \gamma \rightarrow \nu \bar{\nu} \\
& \nu \bar{\nu} \rightarrow \gamma \gamma
\end{aligned}
$$

Nevertheless, the cross section for such reactions is too small. In fact, because of the Yang theorem [1], which forbids two photons to couple in a $J=1$ state, their amplitude is exactly zero to order $G_{F}$ [2] in the standard model (SM) and they are suppressed by powers of $\omega / M_{W}$, where $\omega$ is the center-of-mass energy of the photon and $M_{W}$ the $W$ boson mass. In case of massive neutrinos, a suppression factor $m_{\nu} / M_{W}$ is also present, where $m_{\nu}$ is the neutrino mass. For massless neutrinos, the first non-zero contribution is always of order $1 / M_{W}^{4}$, and the SM cross sections have been shown to be negligibly small in Ref. [3].

On the contrary, five-leg processes involving two neutrinos and three photons, such as

$$
\begin{aligned}
& \gamma \nu \rightarrow \gamma \gamma \nu \\
& \gamma \gamma \rightarrow \gamma \nu \bar{\nu} \\
& \nu \bar{\nu} \rightarrow \gamma \gamma \gamma
\end{aligned}
$$

\footnotetext{
*Email address: abada@mail.cern.ch

${ }^{\dagger}$ Email address: matias@mail.cern.ch

*Email address: pittau@mail.cern.ch.
}

are not constrained by Yang's theorem. Moreover, the extra $\alpha$ in the cross section is compensated by an interchange of the $\omega / M_{W}$ suppression by an $\omega / m_{e}$ enhancement.

Recently, Dicus and Repko derived an effective Lagrangian for five-leg photon-neutrino interactions [4]. They based their derivation on the Euler-Heisenberg Lagrangian [5]. After Fierz rearranging, taking the large mass gauge boson limit, and applying Furry's theorem, they found that the amplitude of the five-leg photon-neutrino process reduces to a four-photon amplitude with one photon field replaced by the neutrino current. Using such a Lagrangian, they calculated processes (4), (5) and (6) for energies below the threshold for $e^{+} e^{-}$pair production, showing that the energy dependence is $\omega^{10}$. By extrapolating this result beyond the range of validity of the effective Lagrangian, the resulting cross sections are of the order of $10^{-52} \mathrm{~cm}^{2}$, for $\omega \sim 1 \mathrm{MeV}$. This is to be compared with process (1), whose cross section is of the order of $10^{-65} \mathrm{~cm}^{2}$, for $\omega \sim 1 \mathrm{MeV}$ [3].

Basing their work on Ref. [4], Harris, Wang and Teplitz [6] investigated to which extent five-leg neutrino processes affect the supernova dynamics, in case the results of Ref. [4] could indeed be extrapolated above $1 \mathrm{MeV}$. They estimated that, in order to fit with the data of SN87A, the cross sections of reaction (4) should have the behavior $10^{-52}(\omega / 1 \mathrm{MeV})^{\sim 8.4} \mathrm{~cm}^{2}$ for $\omega$ of the order of few MeV.

Furthermore, the computation of process (5) in Ref. [4] disagrees with the result obtained in 1963 by Hieu and Shabalin [7].

Given the above scenario, the aim of this work is threefold. First, we would like to demonstrate, step by step, in a somehow pedagogical way, the derivation of the effective theory for inelastic photon-neutrino processes from the effective Euler-Heisenberg theory describing elastic photonphoton interactions. Secondly, we want to settle the question of the disagreement between the calculations of Refs. [4] and [7] for process (5), which is an important energy-loss mechanism in the stellar evolution. The conclusion is that our result agrees with that of Ref. [4]. Thirdly, as a cross-check of Ref. [4], we compute the direct process $\gamma \nu \rightarrow \gamma \gamma \nu$, explicitly giving all polarized cross sections in the effective theory. All calculations in this paper are performed with massless neutrinos.

It goes without saying that computing processes (4), (5) and (6) exactly in the SM, for energies above the $e^{+} e^{-}$ 

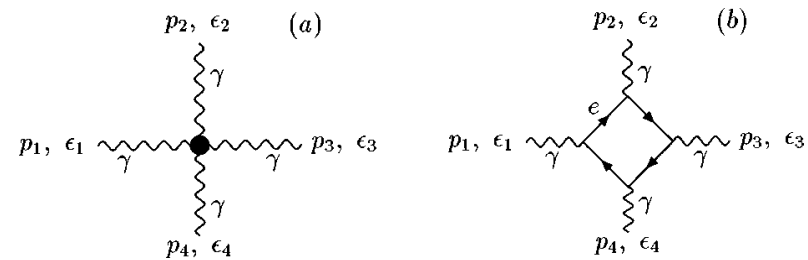

FIG. 1. Four-photon interaction in the effective Lagrangian (a) and in the SM (b).

production threshold, is of extreme interest so as to precisely settle their role in astrophysics. Such a computation is under way [8], and, when completed, will give cross sections valid for all energies up to $\omega<M_{W}$, therefore setting the real range of validity of the effective theory.

The outline of the paper is as follows. In Sec. II, we derive the effective five-leg Lagrangian from the SM. In Sec. III, we compute the polarized and the total cross section for $\gamma \gamma \rightarrow \gamma \nu \bar{\nu}$. Finally, in Sec. IV, we deal with the process $\gamma \nu \rightarrow \gamma \gamma \nu$, whose polarized cross sections are explicitly presented in the Appendix.

\section{DERIVATION OF THE EFFECTIVE LAGRANGIAN}

Our starting point is the leading term of the EulerHeisenberg Lagrangian [5], describing the photon-photon interaction of Fig. 1(a):

$$
\mathcal{L}_{\mathrm{E}-\mathrm{H}}=\frac{\alpha^{2}}{180 m_{e}^{4}}\left[5\left(F_{\mu \nu} F^{\mu \nu}\right)^{2}-14 F_{\mu \nu} F^{\nu \lambda} F_{\lambda \rho} F^{\rho \mu}\right]+\mathcal{O}\left(\alpha^{3}\right) \text {. }
$$

In this equation, $\alpha$ is the QED fine structure constant, $m_{e}$ the electron mass and $F_{\mu \nu}$ the photon field-strength tensor. $\mathcal{L}_{\mathrm{E}-\mathrm{H}}$ can be obtained by matching, in the large $m_{e}$ limit, the exact result with all possible operators compatible with the symmetries, at a given order in the momenta and powers of $1 / M_{W}^{2}$.

In order to find the relation between the effective Lagrangian of Eq. (7) and the one describing processes (4), (5) and (6), we write down the corresponding amplitudes in the SM, and show under which approximations they are equivalent, up to a global factor.

At the one-loop and at leading order in $1 / M_{W}^{2}$, reactions (4), (5) and (6) are given by the diagrams of Fig. 2, plus all possible permutations of the photon legs. The scale is determined by the mass of the fermion running in the loop, so that, at low energies, the leading contribution is given by the
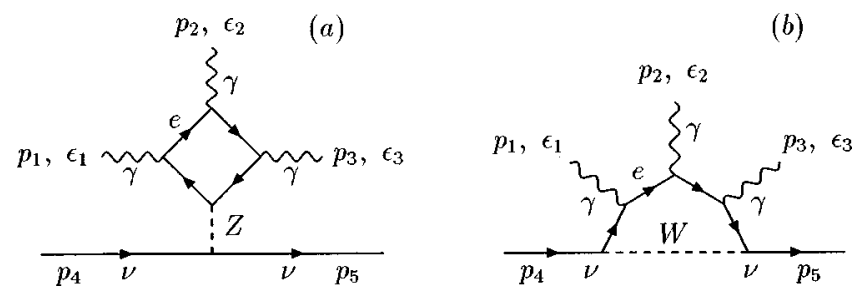

FIG. 2. SM leading diagrams contributing to five-leg photonneutrino processes. mass of the electron. Amplitudes involving higher mass particles, such as $\mu, \tau$, gauge bosons $G$, or hadrons $H$ (at these energies quarks combine into hadrons), are suppressed by powers of $1 / M_{i}$, with $i=\mu, \tau, G, H$. It is precisely the appearance of $m_{e}$ as the scale, instead of $M_{W}$ [which turns out to be the scale that controls reactions (1), (2) and (3)], that makes these processes relevant at energies of the order of few MeV.

We denote by $A_{i j k}$ and $B_{i j k}$ the contributions coming from diagrams (a) and (b) in Fig. 2, respectively. With this notation, $i, j, k$ label a particular permutation of the photon legs. Therefore, the total amplitude reads

$$
\begin{aligned}
\mathcal{A}_{P}^{\mathrm{SM}}= & \epsilon^{\alpha}\left(\vec{P}_{1}, \lambda_{1}\right) \epsilon^{\beta}\left(\vec{P}_{2}, \lambda_{2}\right) \epsilon^{\gamma}\left(\vec{P}_{3}, \lambda_{3}\right) \\
& \times\left[\left(A_{123}^{\alpha \beta \gamma}+A_{321}^{\alpha \beta \gamma}\right)+\left(A_{132}^{\alpha \beta \gamma}+A_{231}^{\alpha \beta \gamma}\right)\right. \\
& +\left(A_{213}^{\alpha \beta \gamma}+A_{312}^{\alpha \beta \gamma}\right)+\left(B_{123}^{\alpha \beta \gamma}+B_{321}^{\alpha \beta \gamma}\right)+\left(B_{132}^{\alpha \beta \gamma}+B_{231}^{\alpha \beta \gamma}\right) \\
& \left.+\left(B_{213}^{\alpha \beta \gamma}+B_{312}^{\alpha \beta \gamma}\right)\right]
\end{aligned}
$$

where $\epsilon^{\alpha}\left(\vec{P}_{i}, \lambda_{i}\right)$ are the polarization vectors associated with the $i$-th photon. We grouped the amplitudes because, as we shall see, it is convenient to consider the diagrams in pairs. For instance, the amplitudes for the first two diagrams are

$$
\begin{aligned}
A_{123}^{\alpha \beta \gamma}= & -\left(g s_{W}\right)^{3}\left(\frac{g}{2 c_{W}}\right)^{2} \\
& \times \sum_{\tau=+,-} v_{e}^{\tau} \Gamma_{\mu}\left(\frac{1}{\Delta_{Z}}\right) \frac{1}{(2 \pi)^{4}} \\
& \times \int d q^{n} \operatorname{Tr}\left[\gamma^{\mu} w^{\tau} \frac{1}{Q_{23}^{-}} \gamma^{\gamma} \frac{1}{Q_{2}^{-}} \gamma^{\beta} \frac{1}{Q_{0}^{-}} \gamma^{\alpha} \frac{1}{Q_{1}^{-}}\right] \\
A_{321}^{\alpha \beta \gamma=} & -\left(g s_{W}\right)^{3}\left(\frac{g}{2 c_{W}}\right)^{2} \\
& \times \sum_{\tau=+,-} v_{e}^{\tau} \Gamma_{\mu}\left(\frac{1}{\Delta_{Z}}\right) \frac{1}{(2 \pi)^{4}} \\
& \times \int d q^{n} \operatorname{Tr}\left[\gamma^{\mu} w^{\tau} \frac{1}{Q_{-1}^{-}} \gamma^{\alpha} \frac{1}{Q_{0}^{-}}\right. \\
& \left.\times \gamma^{\beta} \frac{1}{Q_{-2}^{-}} \gamma^{\gamma} \frac{1}{Q_{-(23)}^{-}}\right],
\end{aligned}
$$

where $Q_{i}^{ \pm}=\Phi_{i} \pm m_{e} \quad\left(Q_{0}=q, \quad Q_{23}=q+p_{2}+p_{3}, \quad Q_{2}=q\right.$ $+p_{2}, Q_{-1}=q-p_{1}$, etc. $), \quad 1 / \Delta_{Z}=1 /\left(\left(p_{4}+p_{5}\right)^{2}-M_{Z}^{2}\right) \sim$ $-1 / M_{Z}^{2}$ and

$$
v_{e}^{ \pm}=v_{e} \pm a_{e}, \quad v_{e}=-\frac{1}{2}+2 s_{W}^{2}, \quad a_{e}=\frac{1}{2} .
$$

Furthermore, $s_{W}$ and $c_{W}$ are the sine and cosine of the Weinberg angle, $w^{ \pm}=\left(1 \pm \gamma_{5}\right) / 2$ and

$$
\Gamma_{\mu}=\bar{v}_{+}(5) \gamma_{\mu} w_{-} u_{-}(4)
$$


By reversing the trace in $A_{321}$, changing $q$ to $-q$ and $m_{e}$ to $-m_{e}$ (only even powers of $m_{e}$ survive) one finds that $A_{321}$ is equal to $A_{123}$ with the replacement $w^{\tau} \rightarrow w^{-\tau}$. Thus, in the sum, the $\gamma_{5}$ contribution cancels in the trace and the pair loses memory of the axial part:

$$
\begin{gathered}
A_{123}^{\alpha \beta \gamma}+A_{321}^{\alpha \beta \gamma}=-2\left(g s_{W}\right)^{3}\left(\frac{g}{2 c_{W}}\right)^{2} v_{e} \Gamma_{\mu} \frac{1}{\Delta_{Z}} L_{1}^{\mu \alpha \beta \gamma} \\
L_{1}^{\mu \alpha \beta \gamma}=\frac{1}{(2 \pi)^{4}} \int d q^{n} \operatorname{Tr}\left[\gamma^{\mu} \frac{1}{Q_{23}^{-}} \gamma^{\gamma} \frac{1}{Q_{2}^{-}} \gamma^{\beta} \frac{1}{Q_{0}^{-}} \gamma^{\alpha} \frac{1}{Q_{1}^{-}}\right] .
\end{gathered}
$$

The same trick can be applied to each pair of diagrams of type $B$. For instance

$$
\begin{aligned}
B_{123}^{\alpha \beta \gamma}+B_{321}^{\alpha \beta \gamma} & =-4\left(g s_{W}\right)^{3}\left(\frac{g}{2 \sqrt{2}}\right)^{2} \Gamma_{\mu} L_{2}^{\mu \alpha \beta \gamma} \\
L_{2}^{\mu \alpha \beta \gamma}= & \frac{1}{(2 \pi)^{4}} \int d q^{n} \operatorname{Tr}\left[\gamma^{\mu} \frac{1}{Q_{23}^{-}} \gamma^{\gamma} \frac{1}{Q_{2}^{-}} \gamma^{\beta} \frac{1}{Q_{0}^{-}} \gamma^{\alpha} \frac{1}{Q_{1}^{-}}\right] \\
& \times \frac{1}{\Delta_{W}(q)},
\end{aligned}
$$

where $\Delta_{W}(q)=\left(q+p_{2}+p_{3}+p_{5}\right)^{2}-M_{W}^{2}$. At this point, to consistently retain only terms $\mathcal{O}\left(1 / M_{W}^{2}\right)$, one has to expand $1 / \Delta_{W}(q)$ as follows:

$$
\frac{1}{\Delta_{W}(q)}=\frac{1}{q^{2}-M_{W}^{2}}-\frac{k^{2}+2 q \cdot k}{\left(q^{2}-M_{W}^{2}\right)\left((q+k)^{2}-M_{W}^{2}\right)},
$$

where $k=p_{2}+p_{3}+p_{5}$. Once introduced in Eq. (12), the first term in the right-hand side (RHS) of Eq. (13) allows the following splitting:

$$
\begin{aligned}
\frac{1}{q^{2}-m_{e}^{2}} \frac{1}{q^{2}-M_{W}^{2}} \sim & -\frac{1}{M_{W}^{2}}\left(\frac{1}{q^{2}-m_{e}^{2}}-\frac{1}{q^{2}-M_{W}^{2}}\right) \\
& +\mathcal{O}\left(1 / M_{W}^{4}\right) .
\end{aligned}
$$

The first term in the previous equation gives an $L_{1}$-type integral, while we have explicitly checked that, after adding the contributions of all $B^{i j k}$, the second term vanishes at order $1 / M_{W}^{2}$.

On the other hand, the second term on the RHS of Eq. (13) is of order $1 / M_{W}^{4}$, and can therefore be neglected. This can be easily seen by splitting again the denominators as shown in Eq. (14). That procedure already generates an overall $1 / M_{W}^{2}$ factor, and what remains is finite and proportional to an extra factor $1 / M_{W}^{2}$.

In conclusion, at leading order in $1 / M_{W}^{2}$, the set of four diagrams (from a total of 12) is always proportional to $L_{1}$ :

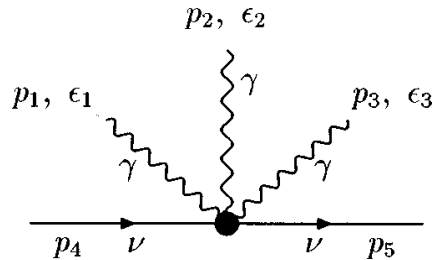

FIG. 3. Five-leg photon-neutrino effective interaction.

$$
\begin{aligned}
A_{123}^{\alpha \beta \gamma}+A_{321}^{\alpha \beta \gamma}+B_{123}^{\alpha \beta \gamma}+B_{321}^{\alpha \beta \gamma}= & -\frac{g^{5} s_{W}^{3}}{2}\left(1+v_{e}\right) \\
& \times \Gamma_{\mu} \frac{1}{\Delta_{Z} c_{W}^{2}} L_{1}^{\mu \alpha \beta \gamma} .
\end{aligned}
$$

Similar results are obtained for the other two groupings of four diagrams, the only difference being a trivial change of momenta and indices inside $L_{1}^{\mu \alpha \beta \gamma}$.

At this point, the correspondence with the four-photon scattering [9] is evident. In fact, by fixing the fourth photon leg and calling $C_{123}^{\alpha \beta \gamma}$ the corresponding amplitude in Fig. $1(\mathrm{~b})$, one finds a contribution proportional to the same integral $L_{1}$ :

$$
C_{123}^{\alpha \beta \gamma}+C_{321}^{\alpha \beta \gamma}=2 g^{4} s_{W}^{4} \epsilon_{\mu}\left(\vec{P}_{4}, \lambda_{4}\right) L_{1}^{\mu \alpha \beta \gamma},
$$

and similar results for the two remaining combinations $C_{132}^{\alpha \beta \gamma}+C_{231}^{\alpha \beta \gamma}$ and $C_{213}^{\alpha \beta \gamma}+C_{312}^{\alpha \beta \gamma}$. Therefore, since the fourphoton process is described, at energies below $m_{e}$, by the Euler-Heisenberg Lagrangian of Eq. (7), it turns out that the same effective Lagrangian can also be used to describe processes (4), (5) and (6). The only change is the replacement of a photon line with a neutrino pair. That can be formally achieved by considering the two neutrinos as a new "gauge field" ${ }^{1} \widetilde{A}_{\nu} \equiv \bar{\psi} \gamma_{\nu}\left(1-\gamma_{5}\right) \psi=2 \Gamma_{\nu}$, with field strength $\widetilde{F}_{\mu \nu}$.

In conclusion, the effective Lagrangian for the five-leg interaction depicted in Fig. 3 reads

$$
\mathcal{L}_{\text {eff }}=\frac{C}{180}\left[5\left(\widetilde{F}_{\mu \nu} F^{\mu \nu}\right)\left(F_{\lambda \rho} F^{\lambda \rho}\right)-14 \widetilde{F}_{\mu \nu} F^{\nu \lambda} F_{\lambda \rho} F^{\rho \mu}\right] .
$$

The constant $C$ remains to be fixed. This can be easily obtained by considering the following ratios of amplitudes in the large- $m_{e}$ limit

$$
\lim _{\text {large } m_{e}} \frac{\mathcal{A}_{4 \gamma}^{\mathrm{SM}}}{\mathcal{A}_{P}^{\mathrm{SM}}}=\frac{\mathcal{A}_{4 \gamma}^{\text {eff }}}{\mathcal{A}_{P}^{\text {eff }}},
$$

where $P$ stands for any of the processes (4), (5) or (6), and $4 \gamma$ is the four-photon interaction.

The first ratio is given by Eqs. (15) and (16):

\footnotetext{
We are using a different convention for $\gamma_{5}$, with respect to Ref.
} 


$$
\frac{\mathcal{A}_{4 \gamma}^{\mathrm{SM}}}{\mathcal{A}_{P}^{\mathrm{SM}}}=\frac{4 M_{W}^{2} s_{W}^{2} \epsilon_{\mu}\left(\vec{P}_{4}, \lambda_{4}\right)}{e\left(1+v_{e}\right) \Gamma_{\mu}},
$$

while the ratio between the two effective amplitudes can be calculated using the effective Lagrangians in Eqs. (7) and (17):

$$
\frac{\mathcal{A}_{4 \gamma}^{\text {eff }}}{\mathcal{A}_{P}^{\text {eff }}}=\frac{e^{4} \epsilon_{\mu}\left(\vec{P}_{4}, \lambda_{4}\right)}{8 \pi^{2} C \Gamma_{\mu}} \frac{1}{m_{e}^{4}},
$$

where we have used the fact that the amplitudes for fourphoton and photon-neutrino processes have exactly the same momentum dependence in both SM and effective theory. Finally, from Eqs. (19) and (20) we derive

$$
C=\frac{g^{5} s_{W}^{3}\left(1+v_{e}\right)}{32 \pi^{2} m_{e}^{4} M_{W}^{2}}=\frac{2 G_{F} \alpha^{3 / 2}\left(1+v_{e}\right)}{\sqrt{2 \pi} m_{e}^{4}},
$$

which agrees with the prefactor of the effective Lagrangian used in Ref. [4].

From the previous derivation, it is clear that the analogy between the two processes also holds at the level of the exact calculations in the SM, within the approximations discussed. Therefore, by substituting

$$
C \rightarrow \frac{\alpha^{2}}{m_{e}^{4}} \quad \text { and } \quad \Gamma_{\mu} \rightarrow 2 \epsilon_{\mu}\left(\vec{P}_{4}, \lambda_{4}\right)
$$

(for any polarization $\lambda_{4}= \pm$ ) in the five-leg photon-neutrino process, the amplitudes of the four-photon process [9] should always be recovered. Notice that in the equivalence between our process $P$ and the four-photon process, one external leg of the four-photon amplitude should be taken off-shell.

The previous derivation can be generalized in a straightforward way to an arbitrary number of external photons.

\section{POLARIZED AND TOTAL CROSS SECTIONS FOR $\gamma \gamma \rightarrow \gamma \nu \bar{\nu}$}

In this section, we give details for the computation of process (5). Our main motivation is the disagreement between the calculations of Refs. [4] and [7]. For massless neutrinos, the differential cross section reads

$$
\begin{aligned}
d \sigma & =\frac{1}{4\left(P_{1} \cdot P_{2}\right)}\left|T_{f i}\right|^{2} d \widetilde{P}_{3} d \widetilde{P}_{\nu} d \widetilde{P}_{\bar{\nu}}(2 \pi)^{4} \delta^{4}\left(P_{i}-P_{f}\right) \\
P_{i} & =P_{1}+P_{2}, \quad P_{f}=P_{3}+P_{\nu}+P_{\bar{\nu}}, \\
d \widetilde{P} & =\frac{d^{3} P}{(2 \pi)^{3} 2 \omega} .
\end{aligned}
$$

Here, $P_{1,2}$ are the momenta of the incoming photons, $P_{3}$ the momentum of the outcoming one and $P_{\nu, \bar{\nu}}$ the momenta of the outcoming neutrino-antineutrino pair. Therefore the dilepton energy is

$$
k_{4} \equiv P_{\nu}+P_{\bar{\nu}}
$$

$T_{f i}$ reads

$$
T_{f i}=C M_{\sigma} \bar{u}_{\nu}\left(P_{\nu}\right) \gamma^{\sigma}\left(1-\gamma_{5}\right) u_{\bar{\nu}}\left(P_{\bar{\nu}}\right),
$$

then

$$
\begin{aligned}
\left|T_{f i}\right|^{2}= & 4 C^{2}\left[\left(P_{\nu} \cdot M\right)\left(P_{\bar{\nu}} \cdot M^{*}\right)+(\nu \leftrightarrow \bar{\nu})-\left(P_{\bar{\nu}} \cdot P_{\nu}\right)|M|^{2}\right. \\
& \left.-i \varepsilon_{\mu \sigma \rho \eta} P_{\nu}^{\mu} P_{\bar{\nu}}^{\sigma} M^{\rho} M^{* \eta}\right]
\end{aligned}
$$

with $C$ given in Eq. (21) and

$$
\begin{aligned}
M_{\sigma} \equiv & \epsilon^{\alpha_{1}}\left(\vec{P}_{1}, \lambda_{1}\right) \epsilon^{\alpha_{2}}\left(\vec{P}_{2}, \lambda_{2}\right) \epsilon^{\alpha_{3}}\left(\vec{P}_{3}, \lambda_{3}\right) \\
& \times M_{\alpha_{1} \alpha_{2} \alpha_{3} \sigma}\left(P_{1}, P_{2}, P_{3}, k_{4}\right) .
\end{aligned}
$$

The functions $\epsilon\left(\vec{P}_{i}, \lambda_{i}\right)$ are the polarization vectors of the photons and $M_{\alpha_{1} \alpha_{2} \alpha_{3} \sigma}$ is the scattering tensor defined by

$$
\begin{aligned}
& M^{\alpha_{1} \alpha_{2} \alpha_{3} \sigma}\left(P_{1}, P_{2}, P_{3}, k_{4}\right) \\
& \quad \equiv U^{\alpha_{1} \beta_{1} \lambda_{1} \alpha_{2} \beta_{2} \lambda_{1} \alpha_{3} \beta_{3} \lambda_{3}} T_{\lambda_{1} \beta_{1} \lambda_{2} \beta_{2} \lambda_{3} \beta_{3} \mu \nu} S^{\mu \nu \rho \sigma} k_{4}^{\rho} .
\end{aligned}
$$

Finally, the tensors $U, T$, and $S$ are

$$
\begin{gathered}
U^{\alpha_{1} \beta_{1} \lambda_{1} \alpha_{2} \beta_{2} \lambda_{2} \alpha_{3} \beta_{3} \lambda_{3}} \equiv\left|\epsilon_{i j k}\right| P_{i}^{\lambda_{1}} P_{j}^{\lambda_{2}} P_{k}^{\lambda_{3}} g^{\alpha_{i} \beta_{1}} g^{\alpha_{j} \beta_{2}} g^{\alpha_{k} \beta_{3}} \\
S_{\mu \nu \rho \sigma} \equiv\left(g_{\mu \rho} g_{\nu \sigma}-(\mu \leftrightarrow \nu)\right)
\end{gathered}
$$

$T^{\lambda_{1} \beta_{1} \lambda_{2} \beta_{2} \lambda_{3} \beta_{3} \mu \nu}$

$$
\begin{aligned}
\equiv & -\frac{10}{180}\left\{\left[g^{\mu \lambda_{1}} g^{\nu \beta_{1}}\left(g^{\lambda_{2} \lambda_{3}} g^{\beta_{2} \beta_{3}}-\left(\lambda_{3} \leftrightarrow \beta_{3}\right)\right)\right]\right. \\
& -[\mu \leftrightarrow \nu]\}+\frac{14}{180}\left\{\left[\left(g^{\lambda_{1} \nu} g^{\beta_{1} \lambda_{2}}-\left(\beta_{1} \leftrightarrow \lambda_{1}\right)\right)\right.\right. \\
& \left.\left.\times\left(g^{\beta_{2} \lambda_{3}} g^{\beta_{3} \mu}-\left(\beta_{3} \leftrightarrow \lambda_{3}\right)\right)\right]-\left[\beta_{2} \leftrightarrow \lambda_{2}\right]\right\} .
\end{aligned}
$$

Using the Ward identity $k_{4}^{\mu} M_{\mu}=0$ and the fact that the last term of Eq. (26) cancels ${ }^{2}$ when taking the square, we simply get

$$
\left|T_{f i}\right|^{2}=-4 C^{2}\left[2\left(P_{\nu} \cdot M\right)\left(P_{\nu} \cdot M^{*}\right)+\left(P_{\bar{\nu}} \cdot P_{\nu}\right)|M|^{2}\right] .
$$

To compute the phase-space integrals, we work in the center-of-mass frame of the two incoming photons:

$$
\begin{gathered}
P_{1}=(\omega, \vec{\omega})=\omega(1, \vec{n}), \quad P_{2}=\omega(1,-\vec{n}), \\
P_{3}=\left(\omega_{1}, \vec{\omega}_{1}\right)=\omega_{1}\left(1, \vec{n}^{\prime}\right), \\
P_{\nu}=\left(\omega_{2}, \vec{\omega}_{2}\right)=\omega_{2}\left(1, \vec{n}^{\prime \prime}\right), \\
P_{\bar{\nu}}=\left(2 \omega-\omega_{1}-\omega_{2},-\vec{\omega}_{1}-\vec{\omega}_{2}\right),
\end{gathered}
$$

\footnotetext{
${ }^{2}$ Since the tensor $M^{\alpha_{1} \alpha_{2} \alpha_{3} \sigma}$ in Eq. (28) is real by construction, the last term of Eq. (26) immediately vanishes, for symmetry reasons, when taking a real representation for the polarization vectors appearing in Eq. (27).
} 
where $\vec{n}, \vec{n}^{\prime}$ and $\vec{n}^{\prime \prime}$ are unit vectors. In this process, $P_{\nu}$ and $P_{\bar{\nu}}$ always appear as a sum in the amplitude, and $k_{4}=(2 \omega$ $\left.-\omega_{1},-\vec{\omega}_{1}\right)$.

The scattering plane is defined by the unit vectors $\vec{n}$ and $\vec{n}^{\prime}$. The polarization vectors of the two photons with momentum $\vec{\omega}$ and $\vec{\omega}_{1}$ can be defined with respect to this plane, as follows:

$$
\begin{aligned}
& \epsilon(\vec{n}, \perp)=\epsilon\left(\vec{n}^{\prime}, \perp\right)=\left(0, \varepsilon_{i j k} \frac{n^{j} n^{\prime k}}{\sin \theta}\right) \\
& \epsilon(\vec{n}, \|)=\left(0, \frac{\vec{n}^{\prime}-\cos \theta \vec{n}}{\sin \theta}\right) \\
& \epsilon\left(\vec{n}^{\prime}, \|\right)=\left(0, \frac{\vec{n}^{\prime} \cos \theta-\vec{n}}{\sin \theta}\right), \quad \cos \theta=\vec{n} \cdot \vec{n}^{\prime} .
\end{aligned}
$$

The completeness and normalization relations are easily checked

$$
\begin{gathered}
\sum_{\lambda=\perp, \|} \epsilon^{\mu}(\vec{k}, \lambda) \epsilon^{\nu}(\vec{k}, \lambda)=-g^{\mu \nu}+\frac{k^{\mu} k^{\prime \nu}+k^{\prime \mu} k^{\nu}}{\left(k \cdot k^{\prime}\right)} \\
\epsilon^{\mu}(\vec{k}, \lambda) \epsilon_{\mu}(\vec{k}, \lambda)=-1, \quad \text { for } \lambda=\perp, \| .
\end{gathered}
$$

In the previous equations, $k=\left(k^{0}, \vec{k}\right)$ is the momentum of the photon and $k^{\prime}$ any arbitrary vector non-parallel to $k$ and such that $k^{\prime 2}=0$. The simplest choice is $k^{\prime} \equiv\left(-k^{0}, \vec{k}\right)$. In the frame defined above, by using the fact that $M$ is real with our choice for the polarization vectors, each of the two terms in Eq. (30) gives a contribution proportional to $|M|^{2}$, for each set of polarizations $\left(\lambda_{1}, \lambda_{2}, \lambda_{3}\right)$. After integrating over the lepton pair, the polarized cross section has the form

$$
\begin{aligned}
d \sigma\left(\lambda_{1}, \lambda_{2}, \lambda_{3}\right)= & \frac{G_{F}^{2} a^{2} \alpha^{3}}{3 m_{e}^{8}\left(P_{1} \cdot P_{2}\right)(2 \pi)^{5}} \\
& \times M^{2}\left(\lambda_{1}, \lambda_{2}, \lambda_{3}\right) k_{4}^{2} \frac{d^{3} P_{3}}{\omega_{1}},
\end{aligned}
$$

where $a=1+v_{e}$. By inserting Eq. (32) in Eq. (34), one explicitly gets

$$
\begin{aligned}
\frac{d \sigma(\perp, \perp, \perp)}{\sin \theta d \theta d \omega_{1}} & =8 \frac{G_{F}^{2} a^{2} \alpha^{3}}{6075 m_{e}^{8} \pi^{4}} \omega^{3} \omega_{1}^{3}\left(\omega-\omega_{1}\right)\left(4 \omega-\sin ^{2} \theta \omega_{1}\right)^{2} \\
\frac{d \sigma((\|,\|, \|))}{\sin \theta d \theta d \omega_{1}}= & 8 \frac{G_{F}^{2} a^{2} \alpha^{3}}{6075 m_{e}^{8} \pi^{4}} \omega^{3} \omega_{1}^{3}\left(\omega-\omega_{1}\right) \\
& \times\left(-4\left(-4+3 \sin ^{2} \theta\right) \omega^{2}\right. \\
& \left.+4 \sin ^{2} \theta \omega \omega_{1}+\sin ^{4} \theta \omega_{1}^{2}\right)
\end{aligned}
$$

$$
\begin{aligned}
& \frac{d \sigma(\perp, \|, \perp)}{\sin \theta d \theta d \omega_{1}}=\frac{G_{F}^{2} a^{2} \alpha^{3}}{48600 m_{e}^{8} \pi^{4}} \omega^{3} \omega_{1}^{3}\left(\omega-\omega_{1}\right) \\
& \times(4(410+374 \cos \theta \\
& \left.-93 \sin ^{2} \theta\right) \omega^{2}-4(79+33 \cos \theta) \\
& \left.\times \sin ^{2} \theta \omega \omega_{1}+9 \sin ^{4} \theta \omega_{1}^{2}\right) \\
& \frac{d \sigma(\perp, \perp, \|)}{\sin \theta d \theta d \omega_{1}}=\frac{G_{F}^{2} a^{2} \alpha^{3}}{12150 m_{e}^{8} \pi^{4}} \omega^{3} \omega_{1}^{3}\left(\omega-\omega_{1}\right) \\
& \times\left(4\left(9+7 \sin ^{2} \theta\right) \omega^{2}-112 \sin ^{2} \theta \omega \omega_{1}\right. \\
& \left.+49 \sin ^{4} \theta \omega_{1}^{2}\right) \\
& \frac{d \sigma(\|,\|, \perp)}{\sin \theta d \theta d \omega_{1}}=\frac{G_{F}^{2} a^{2} \alpha^{3}}{12150 m_{e}^{8} \pi^{4}} \omega^{3} \omega_{1}^{3}\left(\omega-\omega_{1}\right) \\
& \times\left(6 \omega-7 \sin \theta^{2} \omega_{1}\right)^{2} \\
& \frac{d \sigma(\|, \perp,\|)}{\sin \theta d \theta d \omega_{1}}=\frac{G_{F}^{2} a^{2} \alpha^{3}}{48600 m_{e}^{8} \pi^{4}} \omega^{3} \omega_{1}\left(\omega-\omega_{1}\right) \\
& \times\left((34+22 \cos \theta) \omega-3 \sin \theta^{2} \omega_{1}\right)^{2} \\
& \frac{d \sigma(\perp,\|,\|)}{\sin \theta d \theta d \omega_{1}}=\frac{G_{F}^{2} a^{2} \alpha^{3}}{48600 m_{e}^{8} \pi^{4}} \omega^{3} \omega_{1}^{3}\left(\omega-\omega_{1}\right) \\
& \times\left((34-22 \cos \theta) \omega-3 \sin \theta^{2} \omega_{1}\right)^{2} \\
& \frac{d \sigma(\|, \perp, \perp)}{\sin \theta d \theta d \omega_{1}}=\frac{G_{F}^{2} a^{2} \alpha^{3}}{48600 m_{e}^{8} \pi^{4}} \omega^{3} \omega_{1}^{3}\left(\omega-\omega_{1}\right) \\
& \times(4(317-374 \cos \theta \\
& \left.+93 \cos ^{2} \theta\right) \omega^{2}-4\left(79-33 \cos \theta-79 \cos ^{2} \theta\right. \\
& \left.\left.+33 \cos ^{3} \theta\right) \omega \omega_{1}+9 \sin ^{4} \theta \omega_{1}^{2}\right) \text {. }
\end{aligned}
$$

By summing the above contributions and averaging over the initial photon polarizations, one gets the unpolarized differential cross section

$$
\begin{aligned}
\frac{d \sigma}{\sin \theta d \theta d \omega_{1}}= & \frac{G_{F}^{2} a^{2} \alpha^{3}}{48600 m_{e}^{8} \pi^{4}} \omega^{3} \omega_{1}^{3}\left(\omega-\omega_{1}\right) \\
& \times\left(2224 \omega^{2}-592 \sin ^{2} \theta \omega^{2}-520 \sin ^{2} \theta \omega \omega_{1}\right. \\
& \left.+139 \sin ^{4} \theta \omega_{1}^{2}\right)
\end{aligned}
$$

from which the total cross section immediately follows

$$
\sigma(\gamma \gamma \rightarrow \gamma \nu \bar{\nu})=\frac{2144}{637875} \frac{G_{F}^{2} a^{2} \alpha^{3}}{\pi^{4}}\left(\frac{\omega}{m_{e}}\right)^{8} \omega^{2}
$$


The formulas in Eqs. (36) and (37) agree with the results presented in Ref. [4] and disagree with the ones obtained by Hieu and Shabalin [7].

As a cross-check, we computed the polarized amplitudes for the process $\gamma \gamma \rightarrow \gamma \gamma$, using the effective Lagrangian of Eq. (17), where we substituted back the neutrino current with a photon polarization, and put $\omega=\omega_{1}$ in order to have an on-shell photon. We recovered all the polarized amplitudes given in Ref. [9]. ${ }^{3}$

\section{CROSS SECTION FOR $\gamma \nu \rightarrow \gamma \gamma \nu$}

In this section, we present results for the differential and the total cross sections of process (4). As a cross-check, two independent calculations have been performed. On the one hand, we directly squared the amplitude with the help of the completeness relation of Eq. (33). On the other hand, we separately calculated all helicity amplitudes. The corresponding polarized cross sections are given in the appendix. The unpolarized differential cross section reads, in both cases

$$
\begin{aligned}
\frac{d^{2} \sigma}{d \omega_{1} d \omega_{2}}= & \frac{a^{2} \alpha^{3} G_{F}^{2} \omega^{3}}{60750 \pi^{4} m_{e}^{8}}\left(7588 \omega^{5}-21055 \omega^{4}\left(\omega_{1}+\omega_{2}\right)+2 \omega^{3}\left(11063 \omega_{1}^{2}+21750 \omega_{1} \omega_{2}+11063 \omega_{2}^{2}\right)\right. \\
& -2 \omega^{2}\left(6067 \omega_{1}^{3}+15552 \omega_{1}^{2} \omega_{2}+15552 \omega_{1} \omega_{2}^{2}+6067 \omega_{2}^{3}\right)+2 \omega\left(2085 \omega_{1}^{4}+5372 \omega_{1}^{3} \omega_{2}+8103 \omega_{1}^{2} \omega_{2}^{2}\right. \\
& \left.\left.+5372 \omega_{1} \omega_{2}^{3}+2085 \omega_{2}^{4}\right)-139\left(5 \omega_{1}^{5}+15 \omega_{1}^{4} \omega_{2}+26 \omega_{1}^{3} \omega_{2}^{2}+26 \omega_{1}^{2} \omega_{2}^{3}+15 \omega_{1} \omega_{2}^{4}+5 \omega_{2}^{5}\right)\right),
\end{aligned}
$$

where $\omega_{1}$ and $\omega_{2}$ are the final photon energies, computed in the center-of-mass of the initial state. Integrating over $\omega_{1}$ and $\omega_{2}$ gives the total cross section

$$
\sigma(\gamma \nu \rightarrow \gamma \gamma \nu)=\int_{0}^{\omega} d \omega_{1} \int_{\left(\omega-\omega_{1}\right)}^{\omega} d \omega_{2} \frac{d^{2} \sigma}{d \omega_{1} d \omega_{2}}=\frac{262}{127575} \frac{G_{F}^{2} a^{2} \alpha^{3}}{\pi^{4}}\left(\frac{\omega}{m_{e}}\right)^{8} \omega^{2} .
$$

The results in Eqs. (38) and (39) agree with the corresponding expressions in Ref. [4], which we confirm.

\section{CONCLUSIONS}

In this work, we justified the effective Lagrangian approach, based on the four-photon Euler-Heisenberg Lagrangian [5], to compute five-leg photon-neutrino processes. We gave all essential steps for the derivation of the effective Lagrangian at leading order in the Fermi theory. We computed the processes $\gamma \gamma \rightarrow \gamma \nu \bar{\nu}$ and $\gamma \nu \rightarrow \gamma \gamma \nu$, explicitly listing all polarized cross sections.

Concerning the process $\gamma \gamma \rightarrow \gamma \nu \bar{\nu}$, we confirm the results of the calculation reported in Ref. [4], while we disagree with the expressions of Ref. [7].

\section{ACKNOWLEDGMENTS}

We thank S. Bertolini, G. F. Giudice, A. Masiero, and P. Nason for helpful remarks. J.M. acknowledges financial support from a Marie Curie EC Grant (TMR-ERBFMBICT 972147).

\footnotetext{
${ }^{3}$ In [9], the polarized amplitudes are correctly reported, while we found a mistake in the general expression for the four-photon amplitude. The global sign of the fifth line of Eq. (54.21) should be a minus. Since the computation of Ref. [7] is based on Ref. [9], that mistake could be the source of the error in Ref. [7].
}

\section{APPENDIX A: POLARIZED CROSS SECTIONS FOR THE $\gamma \nu \rightarrow \gamma \gamma \nu$ PROCESS}

We work in the center-of-mass frame of the photonneutrino initial state:

$$
\begin{gathered}
P_{1}=(\omega, \vec{\omega})=\omega(1, \vec{n}), \quad P_{2}=\left(\omega_{1}, \vec{\omega}_{1}\right)=\omega_{1}\left(1, \vec{n}^{\prime}\right), \\
P_{3}=\left(\omega_{2}, \vec{\omega}_{2}\right)=\omega_{2}\left(1, \vec{n}^{\prime \prime}\right), \\
P_{4}=(\omega,-\vec{\omega})=\omega(1,-\vec{n}),
\end{gathered}
$$$$
P_{5}=\left(2 \omega-\omega_{1}-\omega_{2},-\vec{\omega}_{1}-\vec{\omega}_{2}\right),
$$

where $\vec{n}, \vec{n}^{\prime}$ and $\vec{n}^{\prime \prime}$ are unit vectors, $P_{1}, P_{2}$ and $P_{3}$ are the initial and the two final photon momenta, respectively, while $P_{4 / 5}$ are the initial/final neutrino momenta. The five independent phase-space variables can be chosen as the two energies $\omega_{1,2}$ and three angles that determine the overall orientation of the tripod $\left(\vec{P}_{2}, \vec{P}_{3}, \vec{P}_{5}\right)$. Two angles $\left(\alpha_{1}, \phi_{1}\right)$ fix the direction of $\vec{P}_{2}$ and one angle $\phi$ takes care of the rotation of the $\left(\vec{P}_{3}, \vec{P}_{5}\right)$ system around $\vec{P}_{2}$. With this choice of variables, the polarization vectors for the incoming photon read 


$$
\begin{gathered}
\epsilon(\vec{n}, \|)=\left(\begin{array}{l}
0 \\
-\sin \phi_{1} \\
-\cos \phi_{1} \cos \alpha_{1} \\
\cos \phi_{1} \sin \alpha_{1}
\end{array}\right), \\
\epsilon(\vec{n}, \perp)=\left(\begin{array}{l}
0 \\
\cos \phi_{1} \\
-\sin \phi_{1} \cos \alpha_{1} \\
\sin \phi_{1} \sin \alpha_{1}
\end{array}\right) .
\end{gathered}
$$

Denoting by $\theta$ the angle between the two final-state photons ( $\cos \theta=\vec{n}^{\prime} \cdot \vec{n}^{\prime \prime}$ ), our choice for the polarization vectors of the two outgoing photons is instead

$$
\begin{aligned}
& \epsilon\left(\vec{n}^{\prime}, \perp\right)=\epsilon\left(\vec{n}^{\prime \prime}, \perp\right)=\left(0, \varepsilon_{i j k} \frac{n^{\prime j} n^{\prime \prime k}}{\sin \theta}\right) \\
& \epsilon\left(\vec{n}^{\prime}, \|\right)=\left(0, \frac{\vec{n}^{\prime \prime}-\cos \theta \vec{n}^{\prime}}{\sin \theta}\right) \\
& \epsilon\left(\vec{n}^{\prime \prime}, \|\right)=\left(0, \frac{\vec{n}^{\prime \prime} \cos \theta-\vec{n}^{\prime}}{\sin \theta}\right)
\end{aligned}
$$

The computation of each set of polarizations gives the following polarized cross sections:

$$
\begin{aligned}
\frac{d^{2} \sigma(\perp, \perp, \perp)}{d \omega_{1} d \omega_{2}}= & \frac{d^{2} \sigma(\|, \perp, \perp)}{d \omega_{1} d \omega_{2}}=\frac{a^{2} \alpha^{3} G_{F}^{2} \omega^{3}}{243000 \pi^{4} m_{e}^{8}}\left(6598 \omega^{5}-365 \omega_{1}^{5}-853 \omega_{1}^{4} \omega_{2}-2822 \omega_{1}^{3} \omega_{2}^{2}-2822 \omega_{1}^{2} \omega_{2}^{3}-853 \omega_{1} \omega_{2}^{4}-365 \omega_{2}^{5}\right. \\
& -14477 \omega^{4}\left(\omega_{1}+\omega_{2}\right)+\omega^{3}\left(10378 \omega_{1}^{2}+28144 \omega_{1} \omega_{2}+10378 \omega_{2}^{2}\right)-2 \omega^{2}\left(2041 \omega_{1}^{3}+8677 \omega_{1}^{2} \omega_{2}+8677 \omega_{1} \omega_{2}^{2}\right. \\
& \left.\left.+2041 \omega_{2}^{3}\right)+4 \omega\left(487 \omega_{1}^{4}+1135 \omega_{1}^{3} \omega_{2}+2858 \omega_{1}^{2} \omega_{2}^{2}+1135 \omega_{1} \omega_{2}^{3}+487 \omega_{2}^{4}\right)\right) \\
\frac{d^{2} \sigma(\|,\|, \|)}{d \omega_{1} d \omega_{2}}= & \frac{d^{2} \sigma(\perp,\|,\|)}{d \omega_{1} d \omega_{2}}=\frac{a^{2} \alpha^{3} G_{F}^{2} \omega^{3}}{243000 \pi^{4} m_{e}^{8}}\left(3342 \omega^{5}-365 \omega_{1}^{5}-677 \omega_{1}^{4} \omega_{2}-1238 \omega_{1}^{3} \omega_{2}^{2}-1238 \omega_{1}^{2} \omega_{2}^{3}-677 \omega_{1} \omega_{2}^{4}-365 \omega_{2}^{5}\right. \\
& -9373 \omega^{4}\left(\omega_{1}+\omega_{2}\right)+\omega^{3}\left(9762 \omega_{1}^{2}+22600 \omega_{1} \omega_{2}+9762 \omega_{2}^{2}\right)-14 \omega^{2}\left(367 \omega_{1}^{3}+1183 \omega_{1}^{2} \omega_{2}+1183 \omega_{1} \omega_{2}^{2}\right. \\
& \left.\left.+367 \omega_{2}^{3}\right)+4 \omega\left(443 \omega_{1}^{4}+1003 \omega_{1}^{3} \omega_{2}+2418 \omega_{1}^{2} \omega_{2}^{2}+1003 \omega_{1} \omega_{2}^{3}+443 \omega_{2}^{4}\right)\right) \\
\frac{d^{2} \sigma(\perp, \|, \perp)}{d \omega_{1} d \omega_{2}}= & \frac{d^{2} \sigma(\|,\|, \perp)}{d \omega_{1} d \omega_{2}}=\frac{a^{2} \alpha^{3} G_{F}^{2} \omega^{3}}{243000 \pi^{4} m_{e}^{8}}\left(10206 \omega^{5}-1025 \omega_{1}^{5}-3273 \omega_{1}^{4} \omega_{2}-5066 \omega_{1}^{3} \omega_{2}^{2}-5330 \omega_{1}^{2} \omega_{2}^{3}-3537 \omega_{1} \omega_{2}^{4}\right. \\
& -1025 \omega_{2}^{5}-\omega^{4}\left(30449 \omega_{1}+29921 \omega_{2}\right)+2 \omega^{3}\left(17289 \omega_{1}^{2}+30814 \omega_{1} \omega_{2}+16893 \omega_{2}^{2}\right)-2 \omega^{2}\left(9829 \omega_{1}^{3}\right. \\
& \left.\left.+22757 \omega_{1}^{2} \omega_{2}+22493 \omega_{1} \omega_{2}^{2}+9829 \omega_{2}^{3}\right)+4 \omega_{1}\left(1587 \omega_{1}^{4}+4270 \omega_{1}^{3} \omega_{2}+5465 \omega_{1}^{2} \omega_{2}^{2}+4336 \omega_{1} \omega_{2}^{3}+1653 \omega_{2}^{4}\right)\right) \\
& \left.\left.+22493 \omega_{1}^{2} \omega_{2}+22757 \omega_{1} \omega_{2}^{2}+9829 \omega_{2}^{3}\right)+4 \omega\left(1653 \omega_{1}^{4}+4336 \omega_{1}^{3} \omega_{2}+5465 \omega_{1}^{2} \omega_{2}^{2}+4270 \omega_{1} \omega_{2}^{3}+1587 \omega_{2}^{4}\right)\right) . \\
& -1025 \omega_{2}^{5}-\omega^{4}\left(29921 \omega_{1}+30449 \omega_{2}\right)+2 \omega^{3}\left(16893 \omega_{1}^{2}+30814 \omega_{1} \omega_{2}+17289 \omega_{2}^{2}\right)-2 \omega^{2}\left(9829 \omega_{1}^{3}\right. \\
& +24 \omega^{2} \sigma(\perp, \perp, \|) \\
d \omega_{1} d \omega_{2} & \frac{d^{2} \sigma(\|, \perp,\|)}{d \omega_{1} d \omega_{2}}=\frac{a^{2} \alpha^{3} G_{F}^{2} \omega^{3}}{243000 \pi^{4} m_{e}^{8}}\left(10206 \omega^{5}-1025 \omega_{1}^{5}-3537 \omega_{1}^{4} \omega_{2}-5330 \omega_{1}^{3} \omega_{2}^{2}-5066 \omega_{1}^{2} \omega_{2}^{3}-3273 \omega_{1} \omega_{2}^{4}\right.
\end{aligned}
$$

[1] C. N. Yang, Phys. Rev. 77, 242 (1950).

[2] M. Gell-Mann, Phys. Rev. Lett. 6, 70 (1961).

[3] D. A. Dicus and W. W. Repko, Phys. Rev. D 48, 5106 (1993).

[4] D. A. Dicus and W. W. Repko, Phys. Rev. Lett. 79, 569 (1997).

[5] H. Euler, Ann. Phys. (Leipzig) 26, 398 (1936); W. Heisenberg and H. Euler, Z. Phys. 98, 714 (1936).

[6] M. Harris, J. Wang, and V. L. Teplitz, “Astrophysical effects of $\nu \gamma \rightarrow \nu \gamma \gamma \gamma$ and its crossed processes," astro-ph/9707113.

[7] N. Van Hieu and E. P. Shabalin, Sov. Phys. JETP 17, 681 (1963).

[8] A. Abada, J. Matias, and R. Pittau, "Direct computation of inelastic photon-neutrino processes in the standard model,', hep-ph/9808294.

[9] A. I. Akhiezer and V. B. Berestetskii, Quantum Electrodynamics (Interscience, New York, 1965). 